\title{
Restricción del crecimiento fetal y trombofilia
}

\author{
Fetal growth restriction and thrombophilia \\ Restrição de crescimento fetal e trombofilia
}

Rosario Moran

ORCID: 0000-0002-4171-8460 Ginecólogo. Ex Asistente Clínica Ginecotocológica B. Hospital de

Clínicas. UdelaR.

Christians Rodríguez

ORCID: 0000-0001-5422-5940

Ginecólogo. Ex Asistente Clínica

Ginecotocológica B. Hospital de

Clínicas. UdelaR.

Florencia Garat

ORCID: 0000-0003-4232-9704

Ginecólogo. Profesor Adjunto

Clínica Ginecotocológica B. Unidad

Ecografía. Hospital de Clínicas.

UdelaR.

Marcelo De Agostini ORCID: 0000-0002-5285-6594 Ginecólogo. Profesor Agregado Clínica Ginecotocológica B. Jefe

Unidad Ecografía. Hospital de Clínicas. UdelaR.
Resumen: La restricción del crecimiento fetal es una patología obstétrica frecuente, multifactorial, con elevada morbimortalidad perinatal, que debe ser abordada por un equipo multidisciplinario. Se define como la incapacidad para lograr el máximo potencial de crecimiento fetal. En Uruguay se recomienda para los criterios diagnósticos los establecidos en 2016 por la International Society of Ultrasound in Obstetric and Gynecology (ISUOG), destacando que la realización del seguimiento del crecimiento ecográfico no debe realizarse en un plazo menor a 2 semanas. Se describen causas maternas, fetales y ovulares Dentro de las probables causas maternas se describen en la literatura las trombofilias, fundamentalmente las adquiridas.

Palabras clave: restricción del crecimiento fetal, trombofilia.

Abstract: Fetal growth restriction is a frequent, multifactorial obstetric pathology with high perinatal morbidity and mortality, which must be addressed by a multidisciplinary team. It is defined as the inability to achieve the maximum potential for fetal growth. In Uruguay, the diagnostic criteria established in 2016 by the International Society of Ultrasound in Obstetric and Gynecology (ISUOG) are recommended for diagnostic criteria, highlighting that ultrasound growth monitoring should not be performed in less than 2 weeks. Maternal, fetal and ovular causes are described. Among the probable maternal causes, thrombophilias, mainly acquired ones, are described in the literature.

Key words: fetal growth restriction, thrombophilia.

Resumo: A restrição do crescimento fetal é uma patologia obstétrica multifatorial frequente, com elevada morbimortalidade perinatal, que deve ser tratada por equipe multidisciplinar. É definida como a incapacidade de atingir o potencial máximo de crescimento fetal. No Uruguai, os critérios diagnósticos estabelecidos em 2016 pela Sociedade Internacional de Ultrassom em Obstetrícia e Ginecologia (ISUOG) são recomendados como critérios diagnósticos, destacandose que o monitoramento ultrassonográfico do crescimento não deve ser realizado em menos de 2 semanas. São descritas as causas maternas, fetais e ovulares, e dentre as prováveis causas maternas, as trombofilias, principalmente adquiridas, são descritas na literatura.

Palavras-chave: restrição de crescimento fetal, trombofilia. 


\section{Introducción}

En los últimos años la restricción del crecimiento fetal (RCF) ha sufrido algunos cambios conceptuales en cuanto a su definición, diagnóstico, seguimiento y terapéutica ${ }^{1}$. Es una patología obstétrica frecuente, multifactorial, con elevada morbimortalidad perinatal, que debe ser abordada por un equipo multidisciplinario ${ }^{1,2}$.

Clásicamente se entiende por RCF a la incapacidad para lograr el máximo potencial de crecimiento fetal. El crecimiento fetal normal es aquel que resulta de una división y crecimiento celular sin interferencias y da como producto final un recién nacido de término, en el cual se ha expresado totalmente su máximo potencial genético'. El crecimiento fetal normal se basa en la comparación de las medidas antropométricas del recién nacido problema, con los estándares obtenidos de neonatos que se consideran sanos para una población determinada resultado de un embarazo sin patologías ${ }^{1}$. El crecimiento fetal está determinado por factores maternos, fetales y placentarios. Esta definición por si misma trae dificultades dado que resulta poco factible conocer el máximo potencial genético fetal y su máximo potencial intrínseco ${ }^{1}$. A esto se suma que para diferentes regiones, países o sociedades científicas la definición de RCF es variable, no existiendo un criterio universal, lo cual genera amplias variaciones en su incidencia, desde un $5 \%$ hasta un $17 \%$ según los diferentes autores ${ }^{2}$.

En Latinoamérica, y en nuestro país, con fines clínico-práctico la definición más utilizada por largo tiempo fue la presencia de un feto que presenta un peso por debajo del estimado para su edad gestacional, y que de nacer en ese momento tendrá un peso por debajo del percentil (P) 10 para la edad gestacional, sumado a un perímetro abdominal menor al percentil 5 para la edad gestacional, siendo este el parámetro más afectado, con una sensibilidad del $100 \%$ y especificidad del $88 \%{ }^{1}$.

El pequeño para la edad gestacional (PEG), se definen como aquellos neonatos anatómicamente normales, sin alteraciones hemodinámicas determinadas por el Doppler y con una adecuada velocidad de crecimiento y cuyo peso se encuentra entre los percentiles 3 y 10.

\section{Diagnóstico}

Para establecer el diagnóstico de RCF existen múltiples criterios internacionales.

En algunos centros de referencia internacional como por ejemplo el Clínic de Barcelona se utiliza la siguiente definición de RCF: 1) Peso fetal estimado (PFE) menor al P3 para la edad gestacional; 2) PFE menor al P10 para la edad gestacional acompañado de signos de compromiso fetal (alteraciones de la circulación feto-placentaria identificadas por Doppler).

Sin embargo la International Society of Ultrasound in Obstetric and Gynecology (ISUOG) en 2016, elaboró un consenso considerando como criterios para el diagnóstico los siguientes ${ }^{3}$ :

- PFE y/o perímetro abdominal (PA) por debajo de P3 para la edad gestacional.

- PFE por debajo del P10 para la edad gestacional, acompañado de signos de compromiso fetal (alteraciones de la circulación feto-placentaria identificadas por Doppler, Indice de pulsatilidad (IP) de las arterias uterinas y/o umbilicales por encima de percentil 95, o una relación IP cerebro - placentaria menor al percentil 5.

- Caída mayor a 2 cuartiles en el percentil del PA o el PFE.

En el año 2017 en Uruguay se realizó una actualización de pautas clínicas de Ecografía para el diagnóstico y manejo de obstetricia y ginecología llevada a cabo por la Unidad de Ecografía de la Facultad de Medicina-UdelaR donde se recomienda para el diagnóstico de RCF seguir los criterios diagnósticos de la ISOUG ${ }^{3}$ antes mencionados. Es importante destacar que la realización del seguimiento de crecimiento ecográfico no debe realizarse en un plazo menor a 2 semanas. 


\section{Etiología}

Se describen causas maternas, fetales y ovulares. Tabla1.

\begin{tabular}{|c|c|c|}
\hline Causas & $\begin{array}{l}\text { Etiologia o factores de } \\
\text { riesgo para RCIU }\end{array}$ & Comentarios \\
\hline \multirow[t]{4}{*}{ Fetales } & Factores genéticos & $\begin{array}{l}\text { Representan } 5-20 \% \text { de las causas de RCF. Incluyen: aneuploidías, disomía } \\
\text { uniparental, mutaciones en un gen (ej: IGF1, IGF2, IGF1R), deleciones } \\
\text { parciales o duplicaciones, cromosomas en anillo e imprimación génica } \\
\text { aberrante. }\end{array}$ \\
\hline & Infecciones & $\begin{array}{l}\text { Representan el } 5-10 \% \text {. CMV y Toxoplasmosis son las más frecuentes } \\
\text { relacionadas a RCF en los países desarrollados. Otros virus: rubeola, } \\
\text { varicela-zoster, sífilis y herpes simple. }\end{array}$ \\
\hline & Anomalías estructurales & Anomalías congénitas. A veces coexisten con alteraciones citogenéticas. \\
\hline & Gestación múltiple & \\
\hline \multirow[t]{5}{*}{ Maternos } & Factores genéticos & $\begin{array}{l}\text { Las mujeres que fueron al nacimiento PEG, tienen dos veces más riesgo } \\
\text { de tener RCF. Las mujeres que tuvieron una RCF en embarazos previos } \\
\text { tienen mayor riesgo en las siguientes gestas. }\end{array}$ \\
\hline & $\begin{array}{l}\text { Comorbilidad médica u } \\
\text { obstétrica }\end{array}$ & $\begin{array}{l}\text { Preeclampsia, abruptio placentario, HTA crónica, ERC, diabetes pre- } \\
\text { gestacional, LES, SAF, cardiopatías, enfermedades respiratorias crónicas, } \\
\text { anemia severa, anemia falciforme, alteraciones uterinas, consumo } \\
\text { alcohol, tabaquismo, consumo de drogas (heroína, cocaína) sangrado } \\
\text { importante en primer trimestre. }\end{array}$ \\
\hline & $\begin{array}{l}\text { Exposición a agentes } \\
\text { teratogénicos }\end{array}$ & $\begin{array}{l}\text { Fámacos: warfarina, anticonvulsivantes (ej: ácido valproico), agentes anti- } \\
\text { neoplásicos, antagonistas del ácido fólico, etc. Estos pueden causar RCF } \\
\text { asociado a malformaciones. Exposición a dosis terapéuticas de radiación } \\
\text { (radioterapia). }\end{array}$ \\
\hline & $\begin{array}{l}\text { Utilización de tecnología de } \\
\text { reproducción asistida }\end{array}$ & $\begin{array}{l}\text { Los embarazos concebidos por reproducción asistida tienen mayor } \\
\text { incidencia de RCF que los concebidos naturalmente. }\end{array}$ \\
\hline & Otros & $\begin{array}{l}\text { Bajo peso molecular, escasa ganancia de peso materno, malabsorción, } \\
\text { pobre estado nutricional, corto período intergenésico, residentes en altas } \\
\text { altitudes, edades maternas extremas. }\end{array}$ \\
\hline \multirow[t]{3}{*}{ Ovulares } & $\begin{array}{l}\text { Mosaicismo placentario } \\
\text { confinado }\end{array}$ & $\begin{array}{l}\text { Hace referencia a la presencia de mosaicismo cromosómico en la } \\
\text { placenta pero no en el feto. La severidad de la RCF depende del tipo de } \\
\text { alteración cromosómica presente, de la proporción de células mosaico y } \\
\text { de la presencia de disomía uniparental. }\end{array}$ \\
\hline & Isquemia placentaria & Pre-eclampsia, abruptio placentario o combinación de los mismos. \\
\hline & $\begin{array}{l}\text { Cordón grueso y } \\
\text { anormalidades } \\
\text { placentarias }\end{array}$ & $\begin{array}{l}\text { Dentro de estas se destacan arteria umbilical única, inserción } \\
\text { velamentosa del cordón umbilical, inserción marginal del cordón, placenta } \\
\text { bilobulada, placenta circunvalada y hemangioma placentario, entre otros. }\end{array}$ \\
\hline
\end{tabular}

Dentro de las probables causas maternas se describen en la literatura las trombofilias, fundamentalmente las adquiridas ${ }^{2,4,5}$.

\section{a) Trombofilia adquirida - síndrome antifosfolipídico}

Si analizamos los criterios diagnósticos clínicos y paraclínicos de trombofilia, el RCF ha sido descrito como una manifestación obstétrica vinculable a SAF; siendo el criterio clínico de SAF la presencia de un parto pretérmino menor a 34 semanas con recién nacido morfológicamente normal y con peso menor al P10 para la edad gestacional. La evidencia científica para este criterio surge de estudios de casos control y cohortes $^{4-7}$.

Diversos estudios han valorado la relación entre RCF y SAF con resultados contradictorios ${ }^{8-11}$. Varios trabajos prospectivos y algunos retrospectivos han evidenciado que la presencia de títulos altos de anticuerpos antifosfolípidos (AAF) se asocia a RCF y parto pretérmino ${ }^{12-14}$. En un estudio de casos y controles se encontró que un $25 \%$ de los nacimientos que presentaron RCF tenía SAF comprado con $7 \%$ o menos de los embarazos con nacimientos normales ${ }^{14}$. Sin embargo, el número de casos de dicho trabajo es bajo, y otros estudios no han mostrado dicha asociación.

Si bien la evidencia disponible sobre la frecuencia y la magnitud de la asociación entre el SAF y RCF es controversial se recomienda su estudio en pacientes con parto pretérmino menor de 34 semanas con RCF morfológicamente normal. 


\section{b) Trombofilias hereditarias}

Recientes publicaciones demuestran que la asociación entre trombofilia hereditaria (TH) y RCF no tienen un vínculo certero y la evidencia es débil11,15-24. Uno de los trabajos que presenta mayor número de casos y que evalúan la asociación entre RCF y TH fue el meta-análisis de Robertson publicado en 2006 y donde se evidencia ausencia de relación entre las mismas ${ }^{25}$. Factor V Leiden Homocigoto OR 4.64 (IC 95\% 0.19-115.6), Factor V Leiden Heterocigoto OR 2.68 (IC 95\% 0.59-12.13), Factor II G20210A heterocigoto OR 2.92 (IC 95\% 0.62-13.70)25. Dada la pobre asociación entre TH y RCF asociado a la ausencia de un tratamiento efectivo de las $\mathrm{TH}$ en este contexto clínico es que no existe actualmente una recomendación de solicitud de estudios de trombofilia hereditaria en pacientes que presenten el antecedente de $\mathrm{RCF}^{4,26}$.

\section{Recomendaciones}

-Se sugiere la solicitud de AAF en pacientes con el antecedente de un nacimiento de pretérmino menor a 34 semanas con RCF en un recién nacido morfológicamente normal.

-No se ha establecido asociación entre RCF y trombofilia hereditaria por lo que no se recomienda su estudio ni tratamiento (grado de recomendación $3 b$ )

\section{Bibliografía}

1- Fescina RH, De Mucio B, Martínez G, Alemán A, Sosa C, Mainero L, et al. Vigilancia del crecimiento fetal: manual de autoinstrucción. 2a. ed. Montevideo: CLAP/SMR; 2011. (CLAP/SMR. Publicación científica; 1586).

2- Lausman A, Kingdom J; Maternal fetal Medicine Committee. Intrauterine growth restriction: screening, diagnosis, and management. J Obstet Gynaecol Can. 2013 Aug;35(8):741-748. English, French. doi: $10.1016 /$ S1701-2163(15)30865-3.

3- Salomon LJ, Alfirevic Z, Da Silva Costa F, Deter RL, Figueras F, Ghi T, Glanc P, et al. ISUOG Practice Guidelines: ultrasound assessment of fetal biometry and growth. Ultrasound Obstet Gynecol. 2019 Jun;53(6):715-723. doi: 10.1002/uog.20272.

4- American College of Obstetricians and Gynecologists Women's Health Care Physicians. ACOG Practice Bulletin No. 138: Inherited thrombophilias in pregnancy. Obstet Gynecol. 2013 Sep;122(3):70617. doi: 10.1097/01.AOG.0000433981.36184.4e.

5- Committee on Practice Bulletins-Obstetrics, American College of Obstetricians and Gynecologists. Practice Bulletin No. 132: Antiphospholipid syndrome. Obstet Gynecol. 2012 Dec;120(6):1514-21. doi: 10.1097/01.AOG.0000423816.39542.0f

6- Derksen RH, Khamashta MA, Branch DW. Management of the obstetric antiphospholipid syndrome. Arthritis Rheum. 2004 Apr;50(4):1028-39. doi: 10.1002/art.201.

7- Silver RM, Varner MW, Reddy U, Goldenberg R, Pinar H, Conway D, Bukowski R, et al. Work-up of stillbirth: a review of the evidence. Am. J. Obstet. Gynecol. 2007;196(5):433-44.

8- Schreiber K, Hunt BJ. Managing antiphospholipid syndrome in pregnancy. Thromb. Res. 2019;181:S41-S46.

9- Pierangeli SS, Leader B, Barilaro G, Willis R, Branch DW. Acquired and Inherited Thrombophilia Disorders in Pregnancy. Obstet. Gynecol. Clin. North Am. 2011;38(2):271-295.

10- Wu O, Robertson L, Twaddle S, Lowe GD, Clark P, Greaves M, et al. Screening for thrombophilia in high-risk situations: Systematic review and cost-effectiveness analysis. The Thrombosis: Risk and Economic Assessment of Thrombophilia Screening (TREATS) study. Health Technol. Assess. (Rockv). 2006;10(11):1-75.

11- Alfirevic Z, Roberts D, Martlew V. How strong is the association between maternal thrombophilia and adverse pregnancy outcome? A systematic review. Eur. J. Obstet. Gynecol. Reprod. Biol. 2002;101(1):6-14.

12- Cervera R, Serrano R, Pons-Estel GJ, Ceberio-Hualde L, Shoenfeld Y, de Ramón E, et al. Morbidity and mortality in the antiphospholipid syndrome during a 10-year period: a multicentre prospective study of 1000 patients. Ann. Rheum. Dis. 2015;74(6):1011-8.

13- do Prado AD, Piovesan DM, Staub HL, Horta BL. Association of Anticardiolipin Antibodies. Obstet. Gynecol. 2010;116(6):1433-1443. 
14- Clark EA, Silver RM, Branch DW. Do antiphospholipid antibodies cause preeclampsia and HELLP syndrome? Curr Rheumatol Rep. 2007 Jun;9(3):219-25. doi: 10.1007/s11926-007-0035-9.

15- Simcox LE, Ormesher L, Tower C, Greer IA. Thrombophilia and pregnancy complications. Int. J. Mol. Sci. 2015;16(12):28418-28428.

16- Said JM, Higgins JR, Moses EK, et al. Inherited thrombophilia polymorphisms and pregnancy outcomes in nulliparous women. Obstet. Gynecol. 2010;115(1):5-13.

17- Pierangeli SS, Leader B, Barilaro G, Willis R, Branch DW. Acquired and inherited thrombophilia disorders in pregnancy. Obstet. Gynecol. Clin. North Am. 2011;38(2):271-95, x.

18- Wu O, Robertson L, Twaddle S, Clark P, Greaves M, Walker ID, et al. Screening for thrombophilia in high-risk situations: systematic review and cost-effectiveness analysis. The Thrombosis: Risk and Economic Assessment of Thrombophilia Screening (TREATS) study. Health Technol. Assess. 2006;10(11):1-110.

19- Agorastos T, Karavida A, Lambropoulos A, Constantinidis T, Tzitzimikas S, Chrisafi S, et al. Factor $\mathrm{V}$ Leiden and prothrombin G20210A mutations in pregnancies with adverse outcome. J. Matern. Neonatal Med. 2002;12(4):267-273.

20- Martinelli P, Grandone E, Colaizzo D, Paladini D, Sciannamé N, Margaglione M, et al. Familial thrombophilia and the occurrence of fetal growth restriction. Haematologica. 2001;86(4):428-31.

21- Infante-Rivard C, Rivard GE, Yotov WV, Génin E, Guiguet M, Weinberg C, et al. Absence of association of thrombophilia polymorphisms with intrauterine growth restriction. N. Engl. J. Med. 2002;347(1):1925.

22- Dizon-Townson D, Miller C, Sibai B, Spong CY, Thom E, Wendel G Jr, et al. The relationship of the factor $\mathrm{V}$ Leiden mutation and pregnancy outcomes for mother and fetus. Obstet. Gynecol. 2005;106(3):517-24.

23- Brenner B. Thrombophilia and Adverse Pregnancy Outcome. Obstet. Gynecol. Clin. North Am. 2006;33(3):443-456.

24- Facco F, You W, Grobman W. Genetic thrombophilias and intrauterine growth restriction: a metaanalysis. Obstet. Gynecol. 2009;113(6):1206-16.

25- Robertson L, Wu O, Langhorne P, Twaddle S, Clark P, Lowe GD, et al. Thrombophilia in pregnancy: a systematic review. Br. J. Haematol. 2006;132(2):171-96.

26- Bates SM, Greer IA, Middeldorp S, Veenstra DL, Prabulos AM, Vandvik PO. VTE, thrombophilia, antithrombotic therapy, and pregnancy: Antithrombotic Therapy and Prevention of Thrombosis, 9th ed: American College of Chest Physicians Evidence-Based Clinical Practice Guidelines. Chest. 2012 Feb;141(2 Suppl):e691S-e736S. doi: 10.1378/chest.11-2300.

\section{Aporte de cada autor al trabajo}

Rosario Moran: Concepción y diseño del trabajo, recolección de datos, redacción y revisión crítica del manuscrito.

Christians Rodríguez: Concepción y diseño del trabajo, recolección de datos, redacción y revisión crítica del manuscrito.

Florencia Garat: Concepción y diseño del trabajo, recolección de datos, redacción y revisión crítica del manuscrito.

Marcelo De Agostini: Concepción y diseño del trabajo, recolección de datos, redacción y revisión crítica del manuscrito. 